\title{
MPT64 Antigen Simple and Rapid Test for Identification and Discrimination of Mycobacterium tuberculosis Complex from Nontuberculous Mycobacteria
}

\author{
Andra-Cristina BOSTĂNARU ${ }^{*}$, Daniela DICULENCU ${ }^{2}$, Gheorghe SAVUȚA ${ }^{1}$ \\ ${ }^{1}$ Department of Public Health, Faculty of Veterinary Medicine, University of Agriculture Sciences and \\ Veterinary Medicine "Ion Ionescu de la Brad", Iasi, Romania, \\ ${ }^{2}$ Clinical Hospital of Pulmonology Iași; \\ * corresponding author: andrutza_mvet@yahoo.com
}

Bulletin UASVM Veterinary Medicine 71(2) / 2014,

Print ISSN 1843-5270; Electronic ISSN 1843-5378

DOI:10.15835/buasvmcn-vm: 10477

\begin{abstract}
Tuberculosis is a global pandemic and the incidence of nontuberculous mycobacteria (NTM) appears to be increasing worldwide. In this sense it is necessary that laboratories to use a rapid, accurate test, not laborious which does not require biosecurity measures. Molecular tests are accurate method, but is needs special equipments and lots labour, time and not economically. The aim for this study was to characterization the isolated of mycobacteria by immunochromatographic test, SD BIOLINE TB Ag MPT64 for differentiation into Mycobacterium tuberculosis (MTBC) complex and NTM. The immunochromatographic assay was evaluated with four reference strains of mycobacterial species and other clinical isolates of mycobacteria using the conventional methods as the gold standard. The results indicate that Ag MPT64 can be easily used for rapid discrimination between MTBC and NTM to confirm Mycobacterium tuberculosis specifically obtained from early stages of subculture without any technical complexity.
\end{abstract}

Keywords: Ag MPT64, Mycobacterium tuberculosis complex, Nontuberculous mycobacteria

\section{INTRODUCTION}

Tuberculosis is a global problem and the single most common cause of death from any bacterial agent (Laughon, 2007; Park et al., 2009; WHO, 1996). Members of Mycobacterium tuberculosis complex (MTBC), which includes M. tuberculosis, M. bovis, M. africanum, M. canetti and M. microti cause tuberculosis. In recent years, disease caused by nontuberculous mycobacteria (NTM) are increasing (Kanade et al., 2011; Hankya et al., 2011; van Ingen et al., 2009).

Mycobacterium tuberculosis (MTB) and NTM are different clinically, therefore rapid detection, isolation, and discrimination are essential for appropriate management (ATS, 1997; Hasegawa et al., 2002; Park et al., 2009).

Detection of tuberculosis agent like NTM species by culture and microscopic methods is difficult and time consuming (Hatta et al., 2010).
Therefore, the examination of cultured colonies should be able to discriminate between MTB and NTM to confirm MTB specifically (Tohir et al., 2011).

Commercial diagnostic methods employ molecular biological tests to provide quick and specific methods for the identification of MTC, instead false positive results cannot be never excluded and these assays are still costly in terms of specialized equipment, labour and time (Tohir et al., 2011; Wang et al., 2006).

Considering all aspects and alternative methods that are inexpensive, rapid methods are vital for the identification of mycobacteria (Halima et al., 2011).

Recently, MPT64 antigen assays were developed to discriminate between Mycobacterium tuberculosis complex (MTBC) from NTM by immunochromatography test (Halima et al., 2011). 
MPT64 is a mycobacterial protein secreted only by MTBC and has been proven to differentiate MTBC from NTM (Abe et al., 1999; Halima et al., 2011; Hasegawa et al., 2002). The MPT64 gene is well characterized, and the antigen has been studied extensively (Halima et al., 2011; Nakamura et al., 1998; Oettinger et al., 1994). MPT64, a $24 \mathrm{kDa}$ secretory protein, is one of major antigens from tuberculosis (Tohir et al., 2011).

Standard Diagnostics, Inc (SD) (Yongin, Korea) developed the SD BIOLINE TB Ag MPT64 RAPID $^{\circledR}$ test, which is a simple and rapid test using a mouse monoclonal anti-MPT64 antibody that is capable to discriminate between MTC and NTM by immunochromatographic assay (Tohir et al., 2011; Halima et al., 2011).

\section{MATERIALS AND METHODS}

The commercial assay, "SD BIOLINE TB Ag MPT 64 Rapid®”, manufactured by Standard Diagnostics, Seoul, South Korea, based on the principle of immunochromatography test, was used as per the manufacturer's instructions.

It was tested on the cultures identified at the Clinic of Pulmonary Disease and Faculty of Veterinary Medicine in Iasi.

For this study, were used 58 MTBC and 115 NTM strains from humans and 11 MTBC and 9 NTM from animals and birds (Tab. 1) which were compared with conventional culture identification methods as a gold standard.

The results obtained after the immunochromatographic assay with the strains isolated from hens and mammalian carnivores were compared with PCR amplification by IS6110 sequence for detection of Mycobacterium tuberculosis complex and the insertion sequence IS 1245 for identified of Mycobacterium avium.

$\mathrm{H}_{37}$ Ra Mycobacterium tuberculosis strain and Mycobacterium avium subsp. avium, Mycobacterium abscessus and Mycobacterium marinum obtained from Spanish Type Culture Collection (CECT) were used as the standard positive and negative control for identification purpose (Tab. 1).

Strains that were to be tested by the commercial immunochromatographic assay were first subcultured on Lowenstein Jensen (LJ) medium and incubated for growth at $37^{\circ} \mathrm{C}$ for several weeks.

Allcultureswereexamined usingZiehl-Neelsen (ZN) staining to confirm the presence of acid-fast bacilli (AFB). After cultivation in LJ medium were applied to the immunochromatographic assay slide directly.

The entire test procedure was carried out inside a biological safety cabinet class II. The solid culture isolates were scrapped from the solid medium and suspended in $200 \mu \mathrm{l}$ of extraction buffer (SD) provided in the kit and the suspension was added to the sample well.

When it were condensation fluid in the slant tubes, it was taken direct $100 \mu \mathrm{L}$ of condensation fluid and was applied directly to the sample well without using an extraction buffer that was much faster.

Tests were interpreted after 15 minutes from sample application at room temperature.

The presence of both control and test pink colored bands, indicates the test is positive and

Tab.1. Different Mycobacterial isolates tested with SD BIOLINE TB Ag MPT 64 Rapid $^{\circledR}$

\begin{tabular}{lc}
\hline \multicolumn{1}{c}{ Species } & Isolates (n) \\
\hline Mycobacterium tuberculosis complex & \\
\hline Mycobacterium tuberculosis $\mathrm{H}_{33} \mathrm{Ra}$ & 1 \\
\hline Mycobacterium tuberculosis complex (humans) & 58 \\
\hline Mycobacterium tuberculosis complex (mammalian carnivores) & 11 \\
\hline Nontuberculous Mycobacteria & 1 \\
\hline Mycobacterium avium subsp. avium 7407 & 1 \\
\hline Mycobacterium abscessus 8517 & 1 \\
\hline Mycobacterium marinum 7091 & 9 \\
\hline Mycobacterium avium subsp. avium (hens) & 115 \\
\hline Nontuberculous Mycobacteria unidentified (humans) & $\mathbf{1 9 7}$ \\
\hline Total
\end{tabular}


the presence of only the control band indicates negative result for MTBC. The absence of control band after the test was considered invalid.

The negative results after the commercial immunochromatographic test (ITC) at hens were compared by IS 1245 PCR assay for Mycobacterium avium. The positive results at mammalian carnivores after the ITC were compared with IS6110 PCR for Mycobacterium tuberculosis complex (Miller J. et al., 1999).

\section{RESULTS AND DISCUSSIONS}

Sensitivity and specificity of the commercial assay was calculated using the results of conventio- nal phenotypic as a golden standard from the MTBC and NTM isolates.

The reference strain $\left(\mathrm{H}_{37} \mathrm{Ra}\right)$ showed the presence of MPT64 antigen in the test band (Fig. 1). Similar positive bands were obtained in MTBC isolates from humans and animals $(n=69)$ and negative bands were detected for any NTM isolates $(\mathrm{n}=124)$ with rapidly growing from humans and hens and for the NTM type strain $(n=3)$ which indicates a test sensitivity and specificity of $100 \%$ compared with conventional methods (Tab. 2).

The PCR assay was performed on 20 samples, isolated from animals and birds.

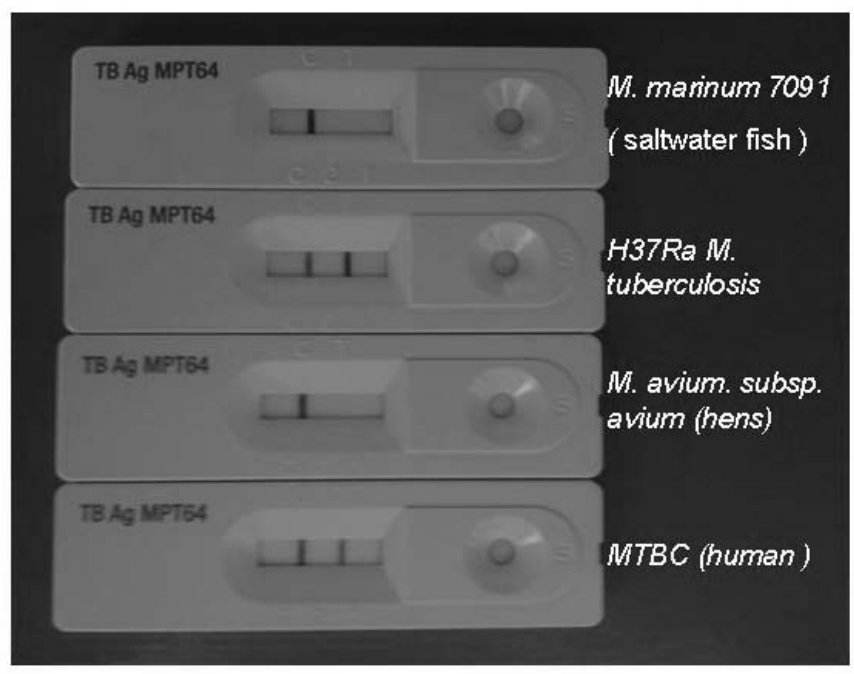

Fig. 1. Identification of the M. tuberculosis complex by the TB Ag MPT 64 kit; strong positive $-\mathrm{H}_{37}$ Ra M. tuberculosis; negative - M. avium subsp. avium

Tab. 2. Sensitivity and Specifity of SD BIOLINE Ag MPT 64

\begin{tabular}{|c|c|c|c|}
\hline Culture method & & \multicolumn{2}{|c|}{ LJ } \\
\hline Test method & & MTBC & NTM \\
\hline \multirow{3}{*}{ Ag MPT 64} & Positive & 70 & 0 \\
\hline & Negative & 0 & 127 \\
\hline & Total & 70 & 127 \\
\hline Sensitivity \& Specifity (\%) & & \multicolumn{2}{|c|}{$100(197 / 197)$} \\
\hline \multirow[t]{2}{*}{ Test method } & \multicolumn{3}{|c|}{ PCR (IS6110/IS1245) } \\
\hline & & MTBC & NTM \\
\hline \multirow[t]{3}{*}{$\begin{array}{l}\text { SD BIOLINE TB } \\
\text { Ag MPT } 64\end{array}$} & Positive & 11 & 0 \\
\hline & Negative & 0 & 9 \\
\hline & Total & 11 & 9 \\
\hline Sensitivity \& Specifity (\%) & \multicolumn{3}{|c|}{$100(20 / 20)$} \\
\hline
\end{tabular}


After the immunochromatographic test, were obtained 9 isolates negatives and 11 isolates positives, this results were confirmed by PCR assay (Tab. 2).

Tests were interpreted in only 15 min after the culture application.

The presence of a control band indicates a negative result, and the presence of two color bands (control and test bands), no matter which band appears first, indicates a positive result. A color band of any intensity was read as a positive result (Fig. 1.).

These results are similar to those observed in other studies in order to assess the sensitivity and specificity of the test (Abe et al., 1999; Fabre et al., 2010; Gaillard et al., 2011; Toihir et al., 2011; Kumar et al., 2011). Ismail et al. (2009), used the same test to demonstrate sensitivity, specificity and positive and negative predictive values of 97\%, 100\%, 100\% and 92\%, respectively. Park et al., (2009) also reported excellent sensitivity (99\%) and specificity (100\%) of the test, with a detection limit by $\left(10^{5} \mathrm{CFU} / \mathrm{mL}\right)$.

The detection limit of this assay is $10^{5} \mathrm{CFU} /$ $\mathrm{ml}$, mentioned and in manufacturer's instructions, which can be prepared with colonies from a solid medium, obtained from early stages of subculture. Therefore, the tuberculosis confirmation can be available with only three colonies from early stage of culture. This shortens the overall time of identification for the clinical isolate by three-four weeks. Both the culture suspension as well as the culture filtrate gave equally satisfactory results.

Instead, the molecular tests requires special equipment and are more expensive for an isolate identification compared with immunochromatographic test and is not able to identify or differentiate the members of MTB complex (Kanade et al., 2012).

This rapid method for confirming MTB culture isolates is reliable, rapid and inexpensive.

\section{CONCLUSION}

In both humans and animals, the immunochromatographic assay it could be a good alternative for a rapid identify MTC from NTM.

Because the optimal management and treatment of a patient with tuberculosis is different from that of patients infected with mycobacteria other than tuberculosis, is necessary to use this rapid and extremely simple test.
“SD BIOLINE TB Ag MPT 64®” assay is a simple, rapid, without any special equipment for protection, economical and accurate test for characterization of clinical mycobacterial isolates suitable for laboratories with poor resource.

Therefore, the workload in order to confirm TB can be reduced remarkably.

Acknowledgments. This work was supported from the Project: "Improvement and Development of Human Resources for Research and Innovation by Doctoral School", Contract: POSDRU - CPP107DMI1/5/S/77222; University of Agricultural Sciences and Veterinary Medicine, Iaşi, Romania.

\section{REFERENCES}

1. Abe C, Hirano K, Tomiyama T (1999). Simple and rapid identification of Mycobacterium tuberculosis complex by immunochromatographic assay using anti-MPB64 monoclonal antibodies. J. Clin. Microbiol. 37:3693-3697.

2. American Thoracic Society (1997). Diagnosis and treatment of disease caused by nontuberculous mycobacteria. Am. J. Respir. Crit. Care Med. 156 (Suppl.):S1-S25.

3. Fabre M, Vong R, Gaillard T, Merens A, Gérome P, SaintBlancard P, Mechaï F, Janvier F, Nouridjan F, Soler C. (2011). Evaluation of the SD BIOLINE TB Ag MPT64 Rapid $((\mathrm{R}))$ for the diagnosis of tuberculosis. Pathol Biol, 59(1), 26, 28.

4. Gaillard T, Fabre M, Martinaud C, Vong R, Brisou P, Soler C (2011). Assessment of the SD Bioline Ag MPT64 Rapid and the MGIT TBc identification tests for the diagnosis of tuberculosis. Diagn Microbiol Infect Dis,70(1), 154, 156.

5. Halima MS, Ismail N, Osman A, Velsmna C, Hoosen AA (2011). Evaluation of TBc Identification Immunochromatographic Assay for Rapid Identification of Mycobacterium tuberculosis Complex in Samples from Broth Cultures. Journal of Clinical Microbiology, p. 19391942 Vol. 49, No. 5.

6. Hasegawa N, Miura T, Ishii K, Yamaguchi K, Lindner TH, Merritt S, Matthews JD, Siddiqi SH (2002). New simple and rapid test for culture confirmation of Mycobacterium tuberculosis complex: a multicenter study. J Clin Microbiol 40: 908-912.

7. Hatta M, Sultan AR, Tandirogang N, Majudi, Yadi (2010). Detection and identification of mycobacteria in sputum from suspected tuberculosis patients. BMC Research Notes, 3:72

8. Ismail NA, Baba K, Pombo D, Hoosen AA (2009). Use of an immunochromatographic kit for the rapid detection of Mycobacterium tuberculosis from broth cultures. Int J Tuberc Lung Dis;13: p. 1045-7.

9. Kanade S, Nataraj G, Suryawanshi R, Mehta P (2012). Utility of MPT 64 antigen detection assay for rapid characterization of mycobacteria in a resource constrained setting. Indian J Tuberc.; 59:92-6. 
10. Kankya C, Muwonge A, Djønne B, Munyeme M, OpudaAsibo J, Skjerve E, Oloya J, Edvardsen V, Johansen TB ( 2011) - Isolation of non-tuberculous mycobacteria from pastoral ecosystems of Uganda: Public Health significance, BMC Public Health, 11:320.

11. Kumar VG, Urs TA, Ranganath RR (2011). MPT 64 Antigen detection for Rapid confirmation of M.tuberculosis isolates. Kumar et al. BMC Research Notes, 4:79.

12. Laughon BE (2007). New tuberculosis drugs in development. Curr. Top. Med. Chem. 7:463-473.

13. Miller JM, Jenny AL, Ellingson JL (1999). Polymerase chain reaction identification of Mycobacterium avium in formalin-fixed, paraffin-embedded animal tissues. J Vet Diagn Invest 11:436-440.

14. Nakamura RM, Velmonte MA, Kawajiri K, Ang CF, Frias RA Mendoza MT, Montoya JC, Honda I, Haga S, Toida I (1998). MPB 64 mycobacterial antigen: a new skin test reagent through patch method for rapid diagnosis of active tuberculosis. Int.J. Tuberc. Lung. Dis. 2:541-546.

15. Oettinger T1, Andersen AB (1994). Cloning and B-cellepitope mapping of MPT64 from Mycobacterium tuberculosis H37Rv. Infect. Immun. 62: 2058-2064.
16. Park MY, Kim JK, Hwang SH, Kim HH, Lee EY, Jeong SH, Chang CL (2009). Evaluation of an immunochromatographic assay kit for rapid identification of Mycobacterium tuberculosis complex in clinical isolates. J. Clin. Microbiol., $47(2), 481,484$.

17. Tohir AH, Rasolofo V, Andrianarisoa SH, Ranjalahy GM, Ramarokoto H (2011). Validation of an immunochromatographic assay kit for the identification of the Mycobacterium tuberculosis complex. Mem Inst Oswaldo Cruz, Rio de Janeiro, Vol. 106(6): 777-780.

18. van Ingen J, Boeree MJ, Dekhuijzen PN, van Soolingen D (2009). Environmental sources of rapid growing nontuberculous mycobacteria causing disease in humans. Clin Microbiol Infect, 15:888-893.

19. Wang JY, Lee LN, Hsu HL, Hsueh PR, Luh KT (2006). Performance assessment of the DR. MTBC Screen assay and the BD ProbeTec ET system for direct detection of Mycobacterium tuberculosis in respiratory specimens. J Clin Microbiol.; 44:716-9.

20. World Health Organization (1996). Report of the tuberculosis epidemic. World Health Organization, Geneva, Switzerland. 